\title{
Synchrony in Sensation
}

\author{
Randy M. Bruno \\ Department of Neuroscience and the Kavli Institute for Brain Science, Columbia University, 701 \\ West 168 ${ }^{\text {th }}$ Street, New York, NY 10032, USA, Phone: 212-305-0181, rb2604@columbia.edu
}

\begin{abstract}
How neurons encode information has been a hotly debated issue. Ultimately, any code must be relevant to the senders, receivers, and connections between them. This review focuses on the transmission of sensory information through the circuit linking thalamus and cortex, two distant brain regions. Strong feedforward inhibition in the thalamocortical circuit renders cortex highly sensitive to the thalamic synchrony evoked by a sensory stimulus. Neuromodulators and feedback connections may modulate the temporal sensitivity of such circuits and gate the propagation of synchrony into other layers and cortical areas. The prevalence of strong feedforward inhibitory circuits throughout the central nervous system suggests that synchrony codes and timing-sensitive circuits may be widespread, occurring well beyond sensory thalamus and cortex.
\end{abstract}

\section{Introduction}

How a message is encoded depends on the medium used to transmit it. In speech from one person's lips to another's ears, text written down on paper, Morse code sent over a cable, or binary code transmitted over a computer network, the same piece of information can be encoded in a myriad of ways. The particular connection between the sender and the receiver is the ultimate determinant of how the sender must encode the message. Similarly, the encoding of neural information-sensations, thoughts, decisions, and memories-is inseparable from the circuits interlinking the brain regions processing that information.

The nature of neural coding has been a contentious and hotly debated issue. Here, I will present the view that substantial progress can be made by considering coding in the context of well-defined local and long-range circuits-where senders, receivers, and their connections are known. This review therefore will focus on communication between thalamus and neocortex, two distant brain regions, and between cortical layers. The cellular make-up, physiological activity, and synaptic connectivity of thalamus and cortex have been extensively characterized for multiple sensory modalities, particularly for barrel cortex, a subregion of rodent primary somatosensory cortex. Individual barrels delineate cortical columns, and these landmarks have greatly facilitated investigation of cortical connectivity and sensory physiology.

In the last 15 years, multiple laboratories have demonstrated that the synchrony of neurons in the whisker-barrel system encodes information. Here, synchrony is defined as correlated discharges of action potentials (APs) from two or more neurons over millisecond timescales. This definition is distinct from correlated neural activity on longer timescales (i.e., tens of

\footnotetext{
(C) 2011 Elsevier Ltd. All rights reserved.

Publisher's Disclaimer: This is a PDF file of an unedited manuscript that has been accepted for publication. As a service to our customers we are providing this early version of the manuscript. The manuscript will undergo copyediting, typesetting, and review of the resulting proof before it is published in its final citable form. Please note that during the production process errors may be discovered which could affect the content, and all legal disclaimers that apply to the journal pertain.
} 
milliseconds to seconds) sometimes employed in behavioral studies. While short-timescale correlations may result from neurons being entrained by local circuit oscillations (e.g., gamma [1]), such potentially interesting interactions with rhythms are beyond this review's scope. The main focus here is millisecond-scale synchrony that emerges when two neurons 1) share a common synaptic input or 2) are embedded in independent circuits whose activity is transiently modulated by the same peripheral stimuli. This review deals with how such synchrony is processed at the network level, not simply integrated by a single neuron.

\section{A common neural circuit, an ideal synchrony detector}

Potent feedforward inhibition (FFI) is a recurring feature of diverse brain areas, including but not limited to neocortex, hippocampus, cerebellum, and amygdala [2-6]. As shown in Fig.1a, the key ingredients of a strong FFI circuit are: 1) a group of pre-synaptic neurons directly excites both the excitatory (spiny, glutamatergic) and inhibitory (aspiny, GABAergic/glycinergic) neurons of a second post-synaptic group but 2) provides greater synaptic input to the inhibitory neurons than excitatory neurons and 3) the post-synaptic neurons are interconnected. Simulations show that circuits lacking inhibition simply relay pre-synaptic activity to post-synaptic neurons (Fig.1b,c). In contrast, post-synaptic neurons in FFI circuits are highly sensitive to the relative timing of APs among pre-synaptic neurons $[7, * 8]$. Rapid, simultaneous increases in discharges of pre-synaptic neurons are able to sufficiently excite the post-synaptic group before excitation is overwhelmed by disynaptic inhibition (Fig.1d). In contrast, slow, temporally uncoordinated changes in pre-synaptic activity elicits fewer or no APs among post-synaptic networks (Fig.1e). Thus, evoked excitation has a limited "window of opportunity" to escape inhibition $[7, * 9,10]$.

Strong FFI is known to exist in the thalamocortical circuit, investigated extensively in the rodent whisker-barrel system. Sensory information from the whiskers initially arrives in cortical layer 4 (L4) via thalamocortical axons. While thalamocortical (TC) axons diverge to contact both excitatory and inhibitory L4 neurons, both in vivo and in vitro studies show that inhibitory neurons receive approximately twice as many synapses and with approximately twice the efficacy [*11,**12]. This differential afferent drive is complemented by strong, convergent inhibitory synapses within L4 [13; Köbl \& Feldmeyer, unpublished results] and faster membrane time constants among inhibitory neurons [**12]. Together, these three mechanisms can ensure that inhibition eventually dominates over excitation.

How can one group of neurons transmit anything to a downstream network through such a suppressive circuit? Individual thalamocortical connections are relatively weak in vivo, depolarizing post-synaptic neurons on average by only $\sim 0.5 \mathrm{mV}$ per thalamic action potential [**14]. Furthermore, in awake animals, at least barrel and auditory cortex operate in low-firing rate regimes $[15,16]$ with neurons spending little time near threshold $[17,18]$. Single afferent neurons alone are, therefore, unlikely to play a major role in the coding and transmission of information at this stage.

Thalamic afferents are, however, highly convergent: an excitatory L4 neuron receives synapses from an estimated $\sim 80-90$ thalamic neurons [*11,**14]. Thalamocortical neurons spontaneously fire APs relatively independently but are tightly time-locked to whisker deflection during sensory stimulation [*9]. Correlated TC discharges derive from the neurons' responding to the same whisker and additionally potent common synaptic inputs. Trigeminal brainstem axons diverge to synapse onto many thalamic neurons, individually eliciting depolarizations of up to $\sim 10 \mathrm{mV}$ in vivo [19]. Consequently, effective sensory stimuli induce transient, strong, millisecond-scale synchrony among TC neurons [**14,20,**21] (see also Fig.2d, left inset), building feedforward excitation fast enough for L4 neurons to reach threshold before being suppressed by FFI. 
Other neocortical sensory circuits appear similarly organized. Assessed functionally, the probability of monosynaptic connections between topographically-aligned thalamic and cortical neurons is similar across visual, auditory, and somatosensory systems [**14,22,23]. Anatomically, 200 spines on a L4 spiny stellate neuron in cat visual cortex receive TC synapses from the lateral geniculate nucleus (LGN) [24], similar to the number of TC contacts inferred for spiny stellate neurons in rat barrel cortex [25]. A direct comparison of LGN convergence onto excitatory and inhibitory neurons is still needed for the visual and auditory systems.

There is, however, clear evidence that some L4 inhibitory neurons in visual cortex have broadly tuned receptive fields, suggesting this subset receives more and/or stronger LGN synapses than excitatory visual cortex neurons do [reviewed in 2]. Consistent with this, visual stimuli evoke strong inhibitory conductances in cat L4 neurons only milliseconds after excitatory conductances [26]. Thus, L4 of visual cortex, like somatosensory L4 [27], possesses a temporal window of opportunity that, along with non-linear integration among excitatory cells, enhances detection of synchronous inputs [26]. Visual thalamus affords such synchronous inputs: LGN cells with overlapping receptive fields exhibit millisecondscale correlations during visual stimuli [*28]. Models incorporating multiple such aspects of visual system organization suggest that simultaneous discharges from several dozen such LGN cells optimally drive cortical spiking [29].

\section{Coding via synchrony}

Thalamic synchrony signals more than the mere presence of a sensory stimulus. The degree of synchrony varies with stimulus features. For instance, velocity of whisker movement affects correlation of extracellularly recorded pairs of thalamic neurons in vivo, even after accounting for overall differences in firing rates [20]. Velocity, which is analogous to contrast in vision, is an important parameter of whisker sensation. As a rodent explores objects, surface friction interacts with the whiskers to produce an irregular pattern of "stickslip" motions [30,31]. Whisker slips across textured surfaces are transient high-velocity movements, and their frequency and velocity vary with surface roughness. The relationship of whisker velocity and neuronal synchrony was initially suggested by the temporally precise responses of sequentially recorded single thalamic units [*9], which further suggested relationships with the exact whisker stimulated, movement direction, the stimulus amplitude, and contact duration [32].

More recently, paired recordings have shown directly that high-frequency repetitive stimuli decrease thalamic synchrony, with important consequences for perception [20,**21]. From the perspective of an ideal observer of spiking activity, cortical neurons detect the presence of stimuli best on the first stimulus presentation (Fig.2a, top). Performance degrades when the stimulus has been presented previously (bottom). However, cortical response magnitudes can discriminate among multiple stimuli (e.g., two velocities) best after cortical cells are adapted by several presentations (Fig.2b). Thus, adaptation degrades detection but enhances discrimination. Prior to adaptation, all stimuli evoke large, easily distinguished cortical spiking responses (Fig.2c, top). After adaptation, spike counts decrease but individual responses cover a larger range (bottom). These trends do not arise trivially from changes in the response magnitudes of thalamic neurons, which can be used to discriminate different stimuli equally well before and after adaptation [**21].

Cortical output instead reflects adaptation in synchrony. As the system adapts to a train of rapidly presented stimuli, the level of thalamic synchrony is reduced (Fig.2d). This degrades detection because stimulus-induced synchrony now falls closer to noise levels and evokes fewer cortical spikes. Although synchrony scales with velocity, the synchrony evoked by 
even relatively low velocities is robust upon first presentation (Fig.2d, colored bars on left), enabling their detection. For repetitive stimuli, however, the synchrony difference between two distinct stimulus types is magnified by adaptation even though the absolute synchrony falls (colored bars on right). Via the strong FFI circuit, the wider range of thalamic synchrony produces a more extensive and therefore discriminable, albeit less detectable, range of cortical spikes. A similar pattern also emerges for multiple directions of whisker movement, suggesting adaptation's effects on synchrony generalize to many stimulus dimensions.

\section{Control points for synchronous activity}

Arousal, motor efference copy, attention, reward anticipation, and other behaviorally relevant non-sensory signals are all thought to impact sensory processing. Does behavior simply change the routing of sensory signals within high-level cognitive areas, or might behavioral state impact sensory processing early on by adjusting communication between regions interconnected in a strong FFI circuit?

An emerging hypothesis is that behavioral state influences dynamics of sensory circuits via neuromodulation [17,**33]. Behavioral state impacts a diverse array of systems that diffusely release neuromodulators, which alter synaptic connections and intrinsic membrane properties. The exact effects depend on connection or cell type or laminar location [**33,34]. For example, a given neuromodulator might have opposite effects on excitatory and inhibitory cells. Such target specificity could tune circuit dynamics rather than simply enhancing or suppressing neurons' overall spike output.

The effects of acetylcholine (ACh), widely suspected of enhancing processing during attentive states, has been perhaps the most studied in a circuit context (Fig.3). In at least the somatosensory and visual systems, thalamocortical terminals onto excitatory, but not inhibitory, neurons possess nicotonic acetylcholine receptors [**33,35,36]. In slice experiments, nicotinic receptors raise release probability at the subset of TC synapses that target excitatory neurons [**33,35], strengthening feedforward excitation relative to FFI. Via muscarinic receptors located on terminals contacting excitatory L4 neurons, ACh additionally decreases intracortical inhibition [**33] and concomitantly recurrent excitation [34]. These cholinergic effects can enhance the circuit's sensitivity to stimuli that evoke low input synchrony. Consistent with this idea, in vivo recordings in both the visual and somatosensory systems show that $\mathrm{ACh}$ lowers the contrast threshold and increases the gain of L4 neurons $[17,36]$.

Transmission in FFI circuits could also be enhanced by modulating the timing and magnitude of input activity. Primary sensory nuclei in thalamus are subject to not only neuromodulation but also tremendous corticothalamic feedback from L6 [37]. In the somatosensory system of sedated rats, pharmacological activation of L6 increases the responsiveness of topographically-aligned thalamocortical neurons to sensory stimuli [38]. Theoretically, such feedback can shift the gains of their targets, sensitizing or desensitizing them to sensory stimuli by altering the balance of excitation and inhibition [**39]. The corticothalamic projection may be engaged by connections from motor cortex, which when pharmacologically activated enhances responses of L6 and subsequently thalamus [40]. In behaving rats, whisking increases thalamic local field potential responses to brainstem input, suggesting motor enhancement of magnitude and/or timing of thalamic responses [40]. Further work is needed to determine whether state directly modulates thalamic synchrony. 


\section{Does synchrony propagate?}

Are these phenomena unique to the thalamocortical system, or do they represent a common scheme for interaction between any two neuronal populations (i.e., two brain regions, cortical columns, or layers)? If synchrony-sensitive circuits are the norm, thalamic-L4 circuitry should be repeated at higher cortical stages. For example, L4 neurons should now act as synchronous inputs to L2/3 in an FFI arrangement. Physiological and anatomical evidence for such exists.

Multiple groups, recording simultaneously from pairs of neurons, have found that sensory stimulation synchronizes L4 neurons in both anesthetized and sedated (unanesthetized) rats [41,42]. Sensory-evoked responses of L4 neurons covary trial-by-trial to a similar degree as with thalamic pairs [41]. Millisecond-wide peaks are clearly visible in cross-correlation analyses of neighboring ( $300 \mu \mathrm{m}$ apart) L4 neurons [42; Ramirez \& Bruno, unpublished results]. Simultaneous monitoring of whisker motion and neural activity in awake behaving rats shows that synchrony not only exists in L4 but also is proportional to the roughness of surfaces palpated by the whiskers [*43]. Rather than degrading or dying off, synchrony appears to cross from thalamus into cortex.

The timing-sensitive architecture of the thalamocortical circuit appears effectively recapitulated for L4-L2/3 connectivity. L4 spiny stellate axons densely innervate the directly overlying region of L2/3 [44]. Paired intracellular recordings in vitro show that excitatory L4 cell synapses onto L2/3 interneurons are approximately twice as efficacious as those onto L2/3 pyramidal neurons [45]. This nearly two-fold difference in L4 drive of L2/3 interneurons and pyramidal cells mirrors the two-fold difference in thalamic drive to L4 interneurons and spiny cells $[* 11, * * 12]$, possibly reinstantiating the strong FFI circuit here (Fig.4).

L2/3 pyramidal neurons exhibit lower firing rates than L4 cells in both anesthetized and awake whisking rats [46] but nonetheless retain synchrony. Two-photon calcium imaging reveals that trial-by-trial responses of L2/3 neurons to whisker stimulation substantially covary when located over the same barrel $[47,48]$. While millisecond-timescale analyses of APs are lacking in this system, array recordings from awake primate somatosensory cortex have demonstrated that stimulation of the hand synchronizes L2/3 pyramidal neurons on at least a 10-msec timescale [49]. Furthermore, dual intracellular recordings from awake mice have demonstrated correlations of membrane potentials of L2/3 neurons [50,51]. These subthreshold correlations, prominent when the mouse sits quietly, are reduced by active whisking but reappear with even tighter synchrony when the whisker contacts an object [52]. All of these findings are consistent with L4-L2/3 circuitry propagating synchrony.

Does the same mode of transmission exist between L2/3 and its targets? This question has yet to be tackled because of the long-range nature of $\mathrm{L} 2 / 3$ axons. In addition to arborizing in L2/3, L5 and L6 of their own cortical region, the axons of L2/3 pyramidal neurons in barrel cortex innervate secondary somatosensory cortex, dysgranular zone, motor cortex, and parietal association areas [53,54]. L2/3's downstream impact may be gated. Whisking decorrelates membrane potentials even after the sensory nerve innervating the whiskers is severed [50,51], suggesting that neuromodulators or long-range cortical synapses acting on L2/3 and/or lower levels (e.g., L4, thalamus, brainstem) alter propagation of synchrony up into L2/3 (see Control Points For Synchronous Activity). Transient and tonic inputs may be separately gated: Models suggest that long-range connections, sparse in L4 but common in $\mathrm{L} 2 / 3$, may unbalance excitation and inhibition to alter propagation of tonic but not synchronous inputs $[39, * 55]$. 


\section{Conclusions}

During a sensory stimulus, a wave of synchronous activity travels from thalamus to cortex and subsequently across layers. Synchrony propagates better than asynchrony due to the strong feedforward inhibitory nature of these circuits. Relative timing codes can signal stimulus features that are behaviorally relevant for sensation. Further measurements of synchrony, by imaging or electrophysiology, are sorely needed throughout the nervous system. Ideally, such measurements would ultimately be carried out in awake behaving animals. Experimental and theoretical studies are also needed to determine which brain regions and animal models exhibit low- or high-firing rate regimes and whether high-firing rate regimes can support synchrony codes.

Beyond being transformed by local circuitry, synchrony may be facilitated or suppressed by neuromodulators acting on key circuit elements or by long-range synapses biased to excitatory or inhibitory targets. While investigations of circuit architecture have become common, the strength and convergence/divergence of many connection types remain unknown. Nevertheless, the recurrence of FFI circuits throughout the nervous system already hints that encoding information via synchrony and routing information by timingsensitive networks may be general principles of brain regions well beyond sensory thalamus and cortex.

\section{Highlights}

- Strong feedforward inhibition sensitizes circuits to synchronous inputs.

- Specific stimulus features determine thalamic synchrony and thus cortical activity.

- Neuromodulators and feedback connections adjust circuit sensitivity to synchrony.

- Synchrony codes may be as prevalent in the brain as feedforward inhibitory circuits.

\section{Acknowledgments}

I am grateful to Larry Abbott, Ken Miller, Tony Movshon, and members of my laboratory for fruitful discussions and comments on the manuscript. This work was supported by grants from NIH (NS069679), the Klingenstein Fund, the Rita Allen Foundation, and the Dana Foundation.

\section{References}

1. Cardin JA, Carlen M, Meletis K, Knoblich U, Zhang F, Deisseroth K, Tsai LH, Moore CI. Driving fast-spiking cells induces gamma rhythm and controls sensory responses. Nature. 2009; 459:663667. [PubMed: 19396156]

2. Alonso JM, Swadlow HA. Thalamocortical specificity and the synthesis of sensory cortical receptive fields. Journal of neurophysiology. 2005; 94:26-32. [PubMed: 15985693]

3. Silberberg G. Polysynaptic subcircuits in the neocortex: spatial and temporal diversity. Current opinion in neurobiology. 2008; 18:332-337. [PubMed: 18801433]

4. Lawrence JJ, McBain CJ. Interneuron diversity series: containing the detonation--feedforward inhibition in the CA3 hippocampus. Trends in neurosciences. 2003; 26:631-640. [PubMed: 14585604]

5. Jorntell H, Bengtsson F, Schonewille M, De Zeeuw CI. Cerebellar molecular layer interneuronscomputational properties and roles in learning. Trends in neurosciences. 2010; 33:524-532. [PubMed: 20869126] 
6. Ehrlich I, Humeau Y, Grenier F, Ciocchi S, Herry C, Luthi A. Amygdala inhibitory circuits and the control of fear memory. Neuron. 2009; 62:757-771. [PubMed: 19555645]

7. Pinto DJ, Brumberg JC, Simons DJ, Ermentrout GB. A quantitative population model of whisker barrels: re-examining the Wilson-Cowan equations. Journal of computational neuroscience. 1996; 3:247-264. [PubMed: 8872703]

8. Kremkow J, Perrinet LU, Masson GS, Aertsen A. Functional consequences of correlated excitatory and inhibitory conductances in cortical networks. Journal of computational neuroscience. 2010; 28:579-594. [PubMed: 20490645] . This modeling study illustrates how effective feedforward inhibition may allow synchronous input patterns to propagate stably across multiple groups of neurons (i.e., cortical layers or brain regions).

9. Pinto DJ, Brumberg JC, Simons DJ. Circuit dynamics and coding strategies in rodent somatosensory cortex. J Neurophysiol. 2000; 83:1158-1166. [PubMed: 10712446] . The authors' previous modeling studies [7] suggested that barrel circuitry renders spike output of barrel neurons sensitive to the temporal distribution of thalamic input. Here, the authors show that cortical spike output is highly correlated with thalamic response times and only weakly correlated with thalamic response magnitudes.

10. Gabernet L, Jadhav SP, Feldman DE, Carandini M, Scanziani M. Somatosensory Integration Controlled by Dynamic Thalamocortical Feed-Forward Inhibition. Neuron. 2005; 48:315-327. [PubMed: 16242411]

11. Bruno RM, Simons DJ. Feedforward mechanisms of excitatory and inhibitory cortical receptive fields. J Neurosci. 2002; 22:10966-10975. [PubMed: 12486192] . Via cross-correlation of single units recorded in lightly sedated (unanesthetized) rats, these experiments demonstrate that in vivo TC synapses onto inhibitory neurons in cortical layer 4 are more convergent and effective than those onto excitatory cells. They also show that the size and directionality of receptive fields of cortical neurons, whether excitatory and inhibitory, are determined by TC convergence.

12. Cruikshank SJ, Lewis TJ, Connors BW. Synaptic basis for intense thalamocortical activation of feedforward inhibitory cells in neocortex. Nat Neurosci. 2007; 10:462-468. [PubMed: 17334362] . Using minimal electrical stimulation in mouse slices, these authors directly show that unitary currents evoked in inhibitory cortical neurons by TC synapses are several-fold larger than currents in excitatory cells. They elegantly show that inhibitory and excitatory cells have an even greater difference in non-minimal currents, indicating an additional difference in TC convergence. These differences in synaptic innervation, rather than in passive membrane properties, are shown to account for strong feedforward inhibition.

13. Sun Q, Huguenard JR, Prince DA. Barrel Cortex Microcircuits: Thalamocortical Feedforward Inhibition in Spiny Stellate Cells Is Mediated by a Small Number of Fast-Spiking Interneurons. J Neurosci. 2006; 26:1219-1230. [PubMed: 16436609]

14. Bruno RM, Sakmann B. Cortex is driven by weak but synchronously active thalamocortical synapses. Science. 2006; 312:1622-1627. [PubMed: 16778049] . Using an intracellular version of cross-correlation, this study reveals that thalamocortical synapses are relatively weak, apparently no different from corticocortical synapses. The study further shows that TC synapses overcome weak connection strength by being highly convergent and synchronously active.

15. O'Connor DH, Peron SP, Huber D, Svoboda K. Neural activity in barrel cortex underlying vibrissa-based object localization in mice. Neuron. 2010; 67:1048-1061. [PubMed: 20869600]

16. Hromadka T, Deweese MR, Zador AM. Sparse representation of sounds in the unanesthetized auditory cortex. PLoS biology. 2008; 6:e16. [PubMed: 18232737]

17. Constantinople CM, Bruno RM. Effects and mechanisms of wakefulness on local cortical networks. Neuron. 2011; 69:1061-1068. [PubMed: 21435553]

18. DeWeese MR, Zador AM. Non-Gaussian membrane potential dynamics imply sparse, synchronous activity in auditory cortex. J Neurosci. 2006; 26:12206-12218. [PubMed: 17122045]

19. Deschenes M, Timofeeva E, Lavallee P. The relay of high-frequency sensory signals in the Whisker-to-barreloid pathway. J Neurosci. 2003; 23:6778-6787. [PubMed: 12890771]

20. Temereanca S, Brown EN, Simons DJ. Rapid changes in thalamic firing synchrony during repetitive whisker stimulation. J Neurosci. 2008; 28:11153-11164. [PubMed: 18971458]

21. Wang Q, Webber RM, Stanley GB. Thalamic synchrony and the adaptive gating of information flow to cortex. Nature neuroscience. 2010; 13:1534-1541.. This in vivo study shows that an ideal 
observer of cortical spiking detects stimuli best on the first stimulus presentation but discriminates different stimuli best after multiple presentations. Adaptation, while reducing thalamic synchrony and consequently cortical spike output, magnifies the differences in thalamic synchrony produced by different stimuli.

22. Reid RC, Alonso JM. Specificity of monosynaptic connections from thalamus to visual cortex. Nature. 1995; 378:281-284. [PubMed: 7477347]

23. Miller LM, Escabi MA, Read HL, Schreiner CE. Functional convergence of response properties in the auditory thalamocortical system. Neuron. 2001; 32:151-160. [PubMed: 11604146]

24. da Costa NM, Martin KA. How Thalamus Connects to Spiny Stellate Cells in the Cat's Visual Cortex. Journal of Neuroscience. 2011; 31:2925-2937. [PubMed: 21414914]

25. Meyer HS, Wimmer VC, Hemberger M, Bruno RM, de Kock CP, Frick A, Sakmann B, Helmstaedter M. Cell type-specific thalamic innervation in a column of rat vibrissal cortex. Cerebral cortex. 2010; 20:2287-2303. [PubMed: 20534783]

26. Cardin JA, Kumbhani RD, Contreras D, Palmer LA. Cellular mechanisms of temporal sensitivity in visual cortex neurons. The Journal of neuroscience. 2010; 30:3652-3662. [PubMed: 20219999]

27. Wilent WB, Contreras D. Dynamics of excitation and inhibition underlying stimulus selectivity in rat somatosensory cortex. Nat Neurosci. 2005; 8:1364-1370. [PubMed: 16158064]

28. Alonso JM, Usrey WM, Reid RC. Precisely correlated firing in cells of the lateral geniculate nucleus. Nature. 1996; 383:815-819. [PubMed: 8893005]. Spike trains from pairs of neurons in cat visual thalamus are shown to be correlated on millisecond time scales when their receptive fields are similar.

29. Wang HP, Spencer D, Fellous JM, Sejnowski TJ. Synchrony of thalamocortical inputs maximizes cortical reliability. Science. 2010; 328:106-109. [PubMed: 20360111]

30. Ritt JT, Andermann ML, Moore CI. Embodied information processing: vibrissa mechanics and texture features shape micromotions in actively sensing rats. Neuron. 2008; 57:599-613. [PubMed: 18304488]

31. Wolfe J, Hill DN, Pahlavan S, Drew PJ, Kleinfeld D, Feldman DE. Texture coding in the rat whisker system: slip-stick versus differential resonance. PLoS Biol. 2008; 6:e215. [PubMed: 18752354]

32. Pinto DJ, Hartings JA, Brumberg JC, Simons DJ. Cortical damping: analysis of thalamocortical response transformations in rodent barrel cortex. Cereb Cortex. 2003; 13:33-44. [PubMed: 12466213]

33. Kruglikov I, Rudyc B. Perisomatic GABA release and thalamocortical integration onto neocortical excitatory cells are regulated by neuromodulators. Neuron. 2008; 58:911-924. [PubMed: $18579081]$. Using a combination of minimal stimulation and paired recordings, the authors demonstrate robust neuromodulation of inhibitory synapses in slices of mouse somatosensory cortex.

34. Eggermann E, Feldmeyer D. Cholinergic filtering in the recurrent excitatory microcircuit of cortical layer 4. Proceedings of the National Academy of Sciences of the United States of America. 2009; 106:11753-11758. [PubMed: 19564614]

35. Gil Z, Connors BW, Amitai Y. Differential regulation of neocortical synapses by neuromodulators and activity. Neuron. 1997; 19:679-686. [PubMed: 9331357]

36. Disney AA, Aoki C, Hawken MJ. Gain modulation by nicotine in macaque v1. Neuron. 2007; 56:701-713. [PubMed: 18031686]

37. Jones EG. Synchrony in the interconnected circuitry of the thalamus and cerebral cortex. Annals of the New York Academy of Sciences. 2009; 1157:10-23. [PubMed: 19351352]

38. Temereanca S, Simons DJ. Functional topography of corticothalamic feedback enhances thalamic spatial response tuning in the somatosensory whisker/barrel system. Neuron. 2004; 41:639-651. [PubMed: 14980211]

39. Vogels TP, Abbott LF. Gating multiple signals through detailed balance of excitation and inhibition in spiking networks. Nature neuroscience. 2009; 12:483-491.. This modeling study investigates how transmission of multiple signals can be gated on and off between different groups of neurons by altering the balance of excitation and inhibition. 
40. Lee S, Carvell GE, Simons DJ. Motor modulation of afferent somatosensory circuits. Nat Neurosci. 2008; 11:1430-1438. [PubMed: 19011625]

41. Khatri V, Bruno RM, Simons DJ. Stimulus-specific and stimulus-nonspecific firing synchrony and its modulation by sensory adaptation in the whisker-to-barrel pathway. J Neurophysiol. 2009; 101:2328-2338. [PubMed: 19279146]

42. Ghoshal A, Pouget P, Popescu M, Ebner F. Early bilateral sensory deprivation blocks the development of coincident discharge in rat barrel cortex. Journal of Neuroscience. 2009; 29:2384 2392. [PubMed: 19244514]

43. Jadhav SP, Wolfe J, Feldman DE. Sparse temporal coding of elementary tactile features during active whisker sensation. Nat Neurosci. 2009; 12:792-800. [PubMed: 19430473] . By simultaneously monitoring whisker motion and cortical activity in behaving rats whisking various textures, these authors show that high-velocity whisker movement is a fundamental tactile feature and is represented by sparse, temporally precise, synchronous spiking in barrel cortex.

44. Egger V, Nevian T, Bruno RM. Subcolumnar dendritic and axonal organization of spiny stellate and star pyramid neurons within a barrel in rat somatosensory cortex. Cereb Cortex. 2008; 18:876889. [PubMed: 17656622]

45. Helmstaedter M, Staiger JF, Sakmann B, Feldmeyer D. Efficient recruitment of layer 2/3 interneurons by layer 4 input in single columns of rat somatosensory cortex. Journal of Neuroscience. 2008; 28:8273-8284. [PubMed: 18701690]

46. de Kock CP, Sakmann B. Spiking in primary somatosensory cortex during natural whisking in awake head-restrained rats is cell-type specific. Proceedings of the National Academy of Sciences of the United States of America. 2009; 106:16446-16450. [PubMed: 19805318]

47. Kerr JN, de Kock CP, Greenberg DS, Bruno RM, Sakmann B, Helmchen F. Spatial organization of neuronal population responses in layer 2/3 of rat barrel cortex. J Neurosci. 2007; 27:13316-13328. [PubMed: 18045926]

48. Sato TR, Gray NW, Mainen ZF, Svoboda K. The Functional Microarchitecture of the Mouse Barrel Cortex. PLoS Biol. 2007; 5:e189. [PubMed: 17622195]

49. Reed JL, Pouget P, Qi HX, Zhou Z, Bernard MR, Burish MJ, Haitas J, Bonds AB, Kaas JH. Widespread spatial integration in primary somatosensory cortex. Proceedings of the National Academy of Sciences of the United States of America. 2008; 105:10233-10237. [PubMed: 18632579]

50. Gentet LJ, Avermann M, Matyas F, Staiger JF, Petersen CC. Membrane potential dynamics of GABAergic neurons in the barrel cortex of behaving mice. Neuron. 2010; 65:422-435. [PubMed: 20159454]

51. Poulet JF, Petersen CC. Internal brain state regulates membrane potential synchrony in barrel cortex of behaving mice. Nature. 2008; 454:881-885. [PubMed: 18633351]

52. Crochet S, Poulet JF, Kremer Y, Petersen CC. Synaptic mechanisms underlying sparse coding of active touch. Neuron. 2011; 69:1160-1175. [PubMed: 21435560]

53. Chakrabarti S, Alloway KD. Differential origin of projections from SI barrel cortex to the whisker representations in SII and MI. The Journal of comparative neurology. 2006; 498:624-636. [PubMed: 16917827]

54. Kim U, Ebner FF. Barrels and septa: separate circuits in rat barrels field cortex. The Journal of comparative neurology. 1999; 408:489-505. [PubMed: 10340500]

55. Kremkow J, Aertsen A, Kumar A. Gating of signal propagation in spiking neural networks by balanced and correlated excitation and inhibition. Journal of Neuroscience. 2010; 30:1576015768. [PubMed: 21106815] . This modeling study investigates how transient synchronous inputs and tonic asynchronous inputs can be separately gated in the same circuit. 
a

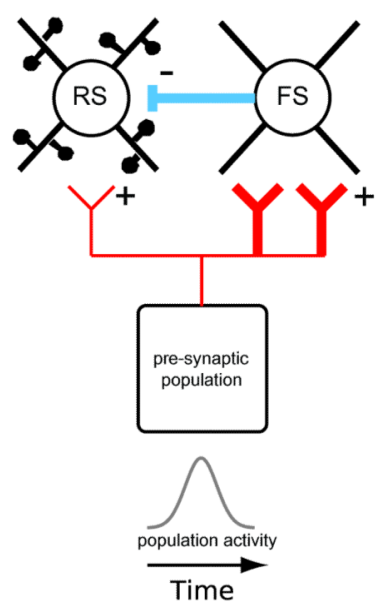

Synchronous

Input

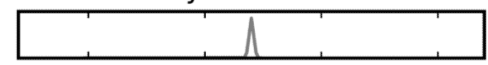

b

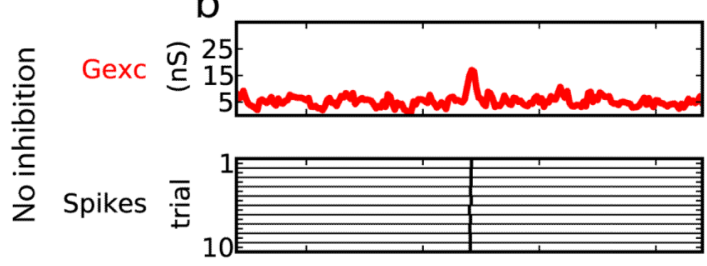

d
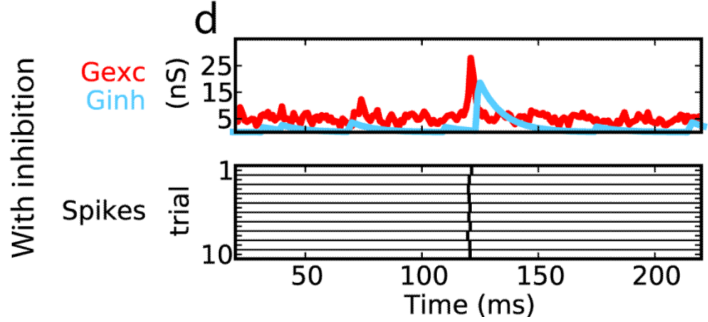

Asynchronous

C

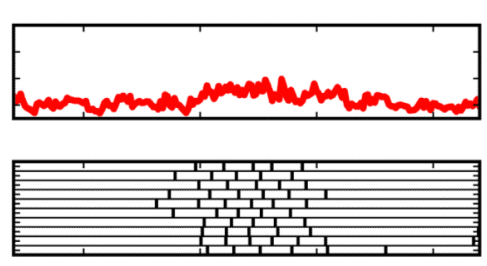

e

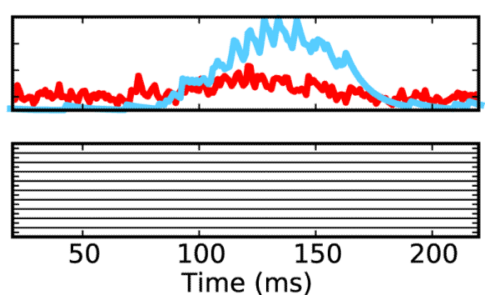

Figure 1.

Circuits with strong feedforward inhibition (FFI) can selectively gate synchronous over asynchronous inputs. (a) Minimal FFI circuit. (b,c) Integration in circuits without FFI and $(d, e)$ with FFI. Shown are the time courses of input population activity (gray) and, for a single post-synaptic excitatory neuron, the total excitatory conductance received (Gexc, red), total inhibitory conductance (Ginh, blue), and spikes output (black). Spike responses are shown for ten consecutive trials whereas conductances are for a single trial. In both the absence and presence of inhibition synchronous input drives post-synaptic output (b,d), but asynchronous inputs do not produce spiking output in the FFI circuit (e). Adapted from [8] with permission from Springer. 

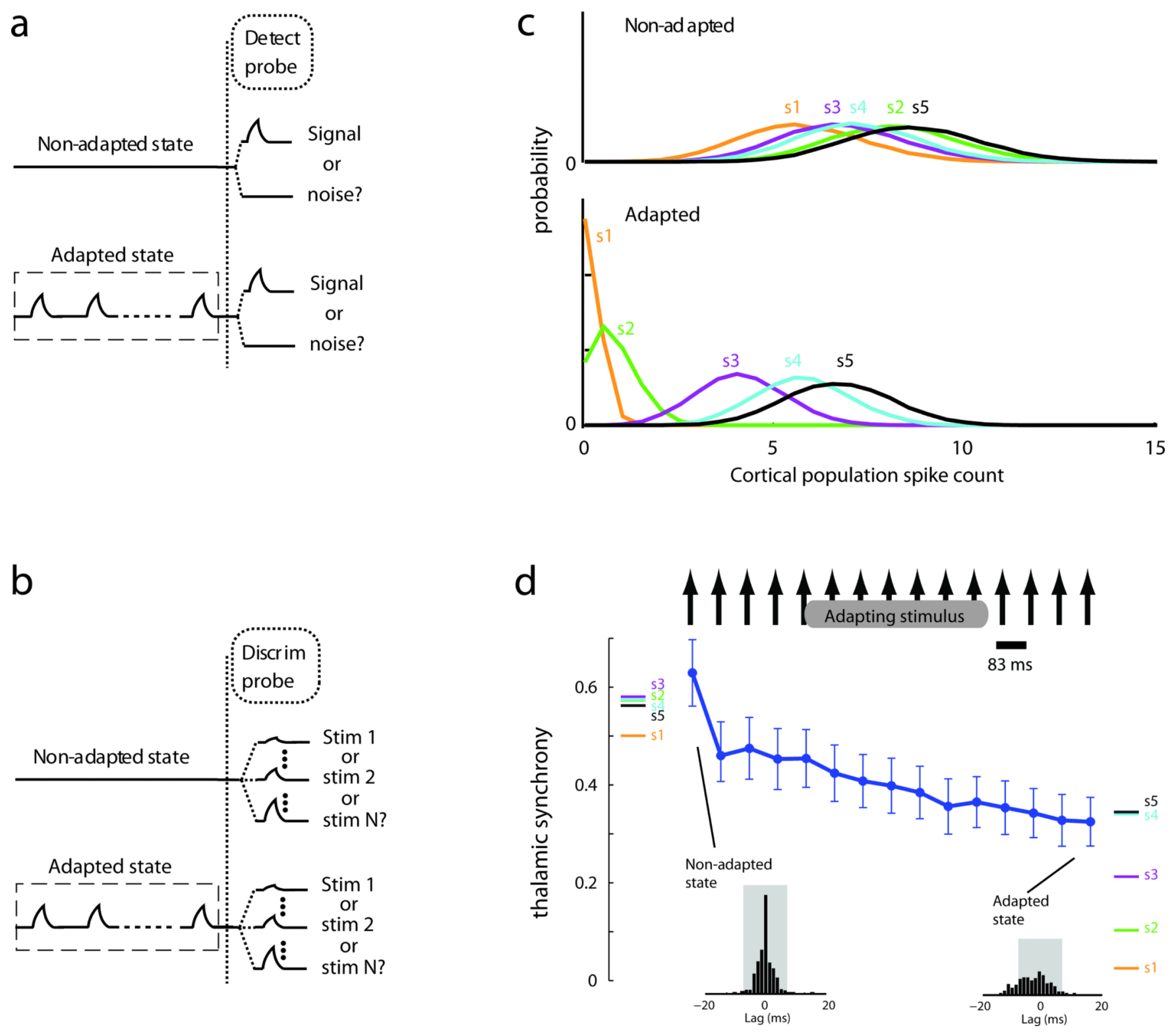

Figure 2.

Adaptation enhances discrimination and degrades detection via thalamic synchrony. (a) Detection analyses of how well an ideal observer can classify the presence/absence of a stimulus following either no preceding stimuli (top) or a train of adapting stimuli (bottom). (b) Discrimination analyses of how well an ideal observer could identify which of multiple stimuli were presented with and without adaptation. (c) Comparison of cortical population responses to 5 different stimuli with (bottom) and without (top) adaptation. (d) Thalamic synchrony, measured by the sum of the normalized cross-correlograms of thalamic pairs (insets) over lags $\pm 7.5 \mathrm{~ms}$, decreases with adaptation but to different degrees for each stimulus, diversifying subsequent cortical responses. Colored bars at left, synchrony evoked by 5 different stimulus velocities without preceding stimuli. Colored bars at right, synchrony evoked by same stimulus set after a train of adapting stimuli. Line, synchrony evoked by each pulse in an adapting train (adapting stimulus is similar to s4 and s5). Adapted from [21] with permission from Macmillan Publishers Ltd. 


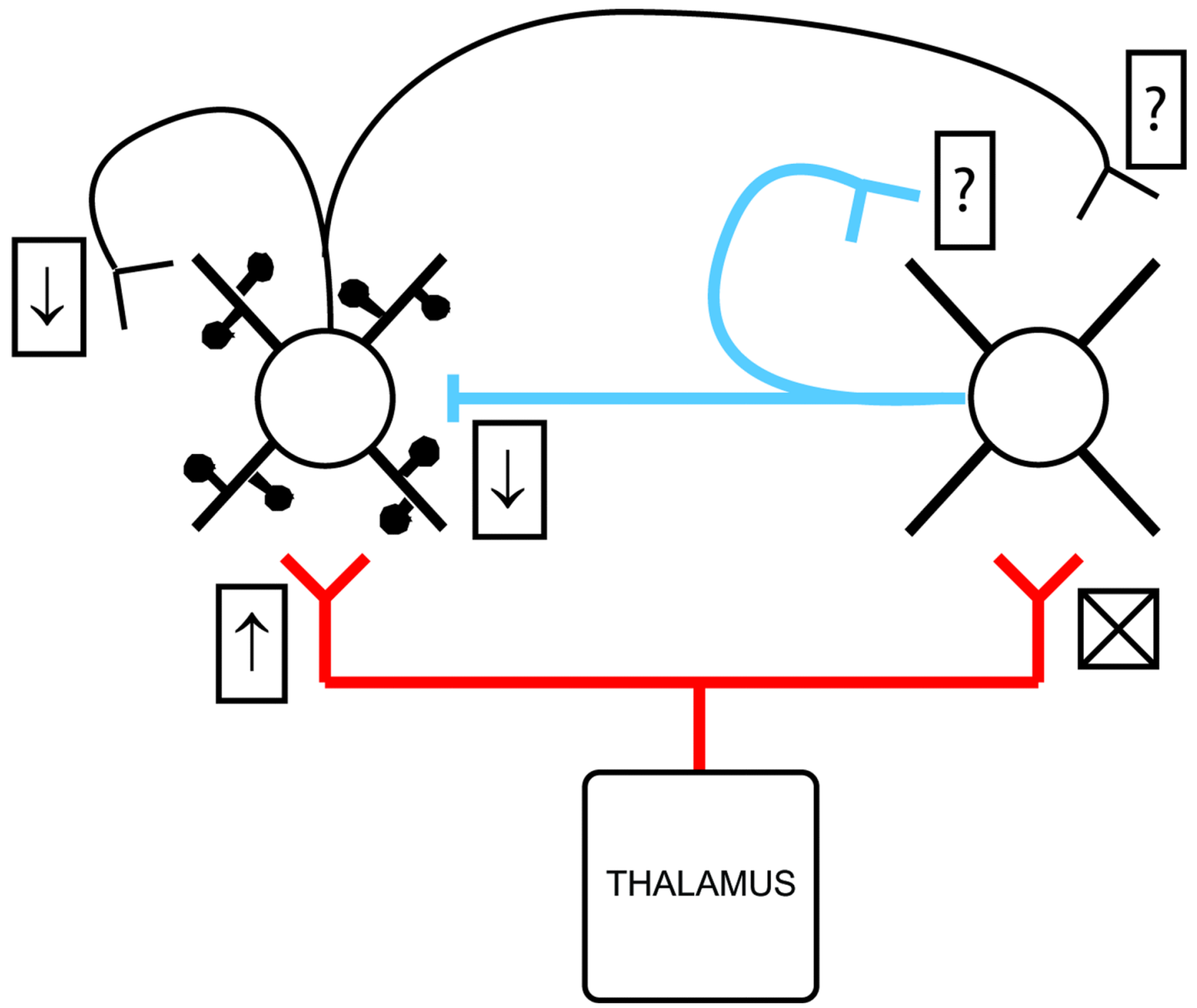

Figure 3.

Effects of cholinergic neuromodulation on synaptic connections may alter L4's sensitivity to synchronous/asynchronous inputs. Acetylcholine increases strength and release probability at TC synapses on L4 excitatory cells via nicotonic receptors ( $\uparrow$ ) but has no action on TC synapses impinging on L4 inhibitory neurons (X). Strength/release is simultaneously decreased at excitatory and inhibitory intracortical synapses onto excitatory L4 neurons ( $\downarrow$ 's). Actions of acetylcholine on synapses contacting inhibitory cells are unknown (?). Effects on intrinsic membrane properties are not illustrated. 
a b

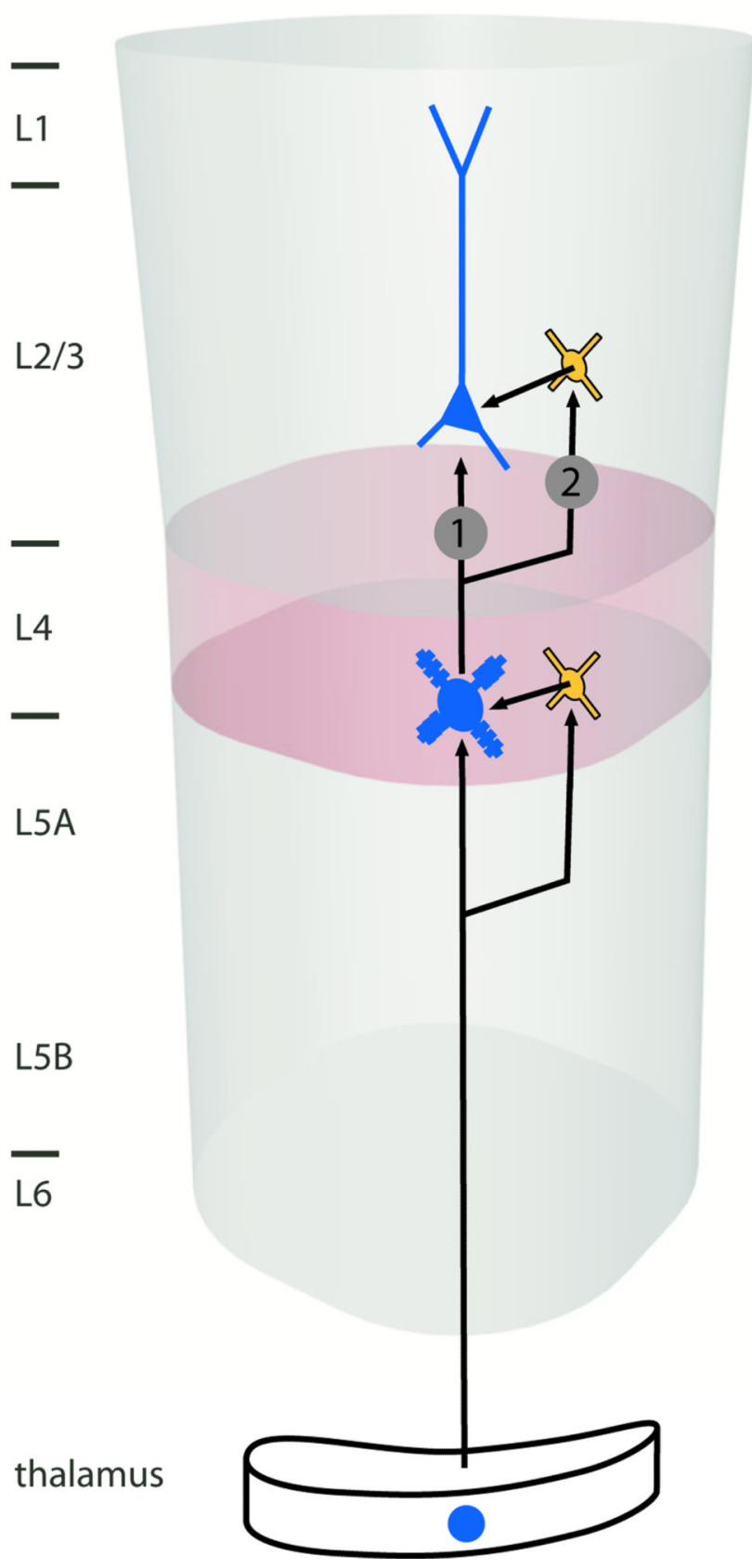

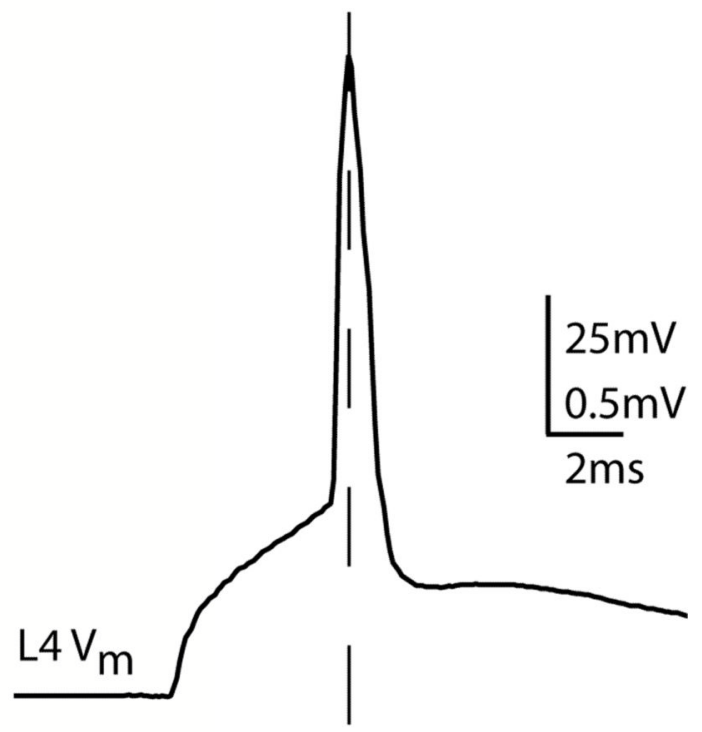

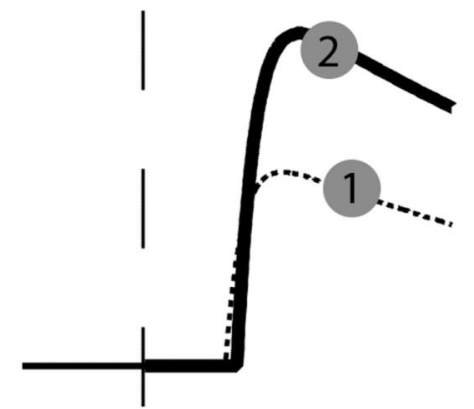

Figure 4.

The L4-L2/3 circuit may, like the thalamic-L4 circuit, contain a strong disynaptic inhibitory pathway. (a) Excitatory connections from thalamus to L4 neurons are paralleled by excitatory connections from L4 to L2/3 interneurons that in turn can inhibit pyramidal neurons in L2/3. (b) An AP in an L4 spiny neuron (top trace) evokes EPSPs in: (1) L2/3 pyramidal cells at a 2-ms latency and small amplitude and (2) L2/3 interneurons at a 2-ms latency and nearly double the amplitude. Adapted from [45] with permission from the Society for Neuroscience. 